\title{
INFLUENCE OF FLOATERS AND POSITIONAL STATUS ON PLAYERS' TACTICAL, PHYSICAL, AND PHYSIOLOGICAL RESPONSES IN SOCCER SMALL-SIDED GAMES
}

original paper

( ) University School of Physical Education in Wroclaw

DOI: https://doi.org/10.5114/hm.2020.91346

\section{GIBSON PRAÇA ${ }^{1}$, GUSTAVO FERNANDES BARBOSA ${ }^{1}$, CRISTIANO MURTA ${ }^{1}$, SARAH DA GLÓRIA TELES BREDT ${ }^{1}$, DANIEL BARREIRA ${ }^{2}$, MAURO HELENO CHAGAS ${ }^{1}$, PABLO JUAN GRECO ${ }^{1}$}

${ }^{1}$ Universidade Federal de Minas Gerais, Belo Horizonte, Brazil

${ }^{2}$ Universidade do Porto, Porto, Portugal

\section{ABSTRACT}

Purpose. Small-sided games (SSG) with floaters are frequently adopted during the training process of soccer players. However, no study has previously investigated how the rules imposed to these additional players impact the physical, tactical, and physiological responses of the regular players. This study aimed to analyse the influence of the number of floaters on players' tactical behaviour, physical demands, and physiological responses in soccer SSGs and to compare these variables between players with different positional statuses.

Methods. Overall, 32 U-13 $(n=16)$ and U-14 ( $n=16)$ players participated in this study, performing four 4-minute 3 vs. 3 SSGs in both 1-floater and 2-floater formats. The frequency of core tactical principles, time-motion variables, and heart rate were used to describe the game multifactorial phenomenon.

Results. Results showed an increased number of tactical actions of delays, defensive coverage, and width and length without ball actions in the 2-floater SSG without differences in physical demands or physiological response. Midfielders presented higher frequencies of penetration and offensive coverage, and lower frequencies of actions of width and length without the ball. There were also higher physical and physiological responses in midfielders.

Conclusions. In conclusion, the 2 -floater 3 vs. 3 format may be an important pedagogical strategy to promote a higher ball circulation pattern during SSGs. Midfielders are the most demanded players tactically, physically, and physiologically in 3 vs. 3 SSGs with floaters.

Key words: soccer, match analysis, small-sided games, tactical principles, physical training, GPS devices

\section{Introduction}

Small-sided games (SSG) are largely used in soccer for technical [1] and tactical [2] training because they allow players to face problem-solving situations in highintensity conditions. For this reason, recent studies have investigated the influence of different SSG formats on players' behaviours by modifying game constraints such as pitch size [3], number of players per team [3, 4], scoring targets [5], and the presence of floaters [6]. Besides, the influence of players' individual characteristics on different performance components during SSG has also been investigated in regard to their level of tactical knowledge [7] and, recently, playing positions [6]. Since both SSG format (e.g. floaters) and positional status (influenced by the players' characteristics) are expected to influence players' performance during SSG, it is important to investigate these aspects simultaneously to provide a deeper understanding on how to use SSG during the training process. The knowledge about the physical demands and physiological responses in different SSG formats may help coaches to better organize the training loads aiming to improve physical skills, such as the aerobic power.

Correspondence address: Gibson Moreira Praça, Universidade Federal de Minas Gerais, Escola de Educação Física, Fisioterapia e Terapia Ocupacional, Departamento de Esportes, Av. Presidente Antônio Carlos, 6627, Campus Pampulha, CEP 31270-901, Belo Horizonte, Minas Gerais, Brazil, e-mail: gibson_moreira@yahoo.com.br

Received: July 8, 2019

Accepted for publication: November 5, 2019

Citation: Praça G, Barbosa GF, Murta C, Bredt SGT, Barreira D, Chagas MH, Greco PJ. Influence of floaters and positional status on players' tactical, physical, and physiological responses in soccer small-sided games. Hum Mov. 2020;21(3):54-63; doi: https://doi.org/10.5114/hm.2020.91346. 
On the other hand, the knowledge about the impact of different formats on tactical behaviour may help coaches to better systematize the use of SSG in accordance with the pedagogical aims of each training session.

Notwithstanding, the literature shows that players' technical performance is influenced by SSG type [1]. In this article, we focused on the influence of floaters during SSG on both physical [8] and tactical aspects $[6,9]$, providing a more integrated perspective of the use of SSG training. It is suggested that the inclusion of these additional players (always to the offensive team, inside or outside the pitch) promotes higher ball circulation, since the usual absence of a defender assigned to this player allows safe passing possibilities [10]. In addition, the presence of floaters is stated to decrease the SSG physical demands in comparison with regular formats [8], indicating a slower pacing strategy to achieve better shooting opportunities and highly coordinated movements between players in defence in order to protect the most important zones of the pitch [11]. In these studies, the floaters were usually adopted in two different ways: in-floater (inside the pitch), as a regular player who changes the team every time ball possession is changed (always in attack) [12], or outfloaters (on the sidelines), as an alternative for a player in ball possession to execute a wall pass [5, 9]. In both cases, the floater can be extensively used for ball progression during the game, since their position is not constrained by any rules of the game and, consequently, they can occupy an in-depth position on the game field. However, the floater in an in-depth position may not represent the reality of the game when the offside rule is adopted, which may reduce the contextual learning transference to the formal match. Therefore, it seems necessary to adopt more adequate rules for the participation of the floaters ahead of the ball line; this has not been previously addressed by the literature.

On the other hand, it seems interesting to adopt rules to keep the floater near the ball carrier to offer support and increase the representativeness of the game with the offside rule. These SSG formats could increase players' ability to keep ball possession and safely progress over the field, which indicates their potential for improving players' ability to successfully build-up the attack. Therefore, the inclusion of two floaters is recommended (one per each team), who must return to their own goal every time the ball possession is lost (which may be achieved by a direct event - interception, tackle, or defensive action of the goalkeeper, for example - or an indirect one - corner kick, goal kick, and free kicks, for example [13]). The influence of these rules on players' behaviours during SSG is still unknown, although their potential pedagogical principles for tactical training can be strongly addressed. It is expected that the 2-floater SSG format (one per each team) would lead to a decrease in both physical and physiological responses in comparison with the traditional 1-floater format, since a build-up attacking style is expected. On the other hand, this attacking style would increase the frequency of actions of width and length in order to allow teams to achieve a full exploration of the pitch size. However, these hypotheses have not been tested yet.

In regard to the influence of players' characteristics on behaviours performed during SSG, the playing positions have been extensively investigated in official soccer matches [14]. In general, it has been demonstrated that midfielders are the most prominent players in building attacks, and also cover higher distances than defenders and forwards in official matches [15]. Few studies examined the influence of playing position on players' performance during SSG, e.g. Praça et al. [6] found similar results in the 3 vs. 3 format and in formal game and, to the best of our knowledge, no studies have explored the influence of playing position on players' behaviour (in both tactical and physical perspectives) during SSG formats with floaters. We hypothesized that midfielders' effective participation would be higher than with the other playing status, leading to increased physical and physiological responses, as well as greater participation in the game centre.

Traditionally, SSG formats have been investigated in a physiological and physical perspective, with the use of heart hate monitors [16] and Global Positioning System (GPS) devices [17]. Although these studies have addressed important issues concerning the use of SSG for physical conditioning, the understanding of its potential tactical improvements has often been neglected. In this context, SSG tactical aspects have been investigated through the use of recently developed tools for the analysis of players' tactical behaviour, such as the system of tactical assessment in soccer (FUT-SAT) [18]. So, to the best of our knowledge, no study has examined the effects of different floater formats on players' behaviour during SSG, especially in accordance with playing positions. Therefore, the aim of this study was to compare the physical and physiological responses, as well as tactical behaviour of soccer players from different positions (defenders, midfielders, and forwards) during SSG with one or two floaters. 


\section{Material and methods}

\section{Participants}

A total of 32 soccer players, $16 \mathrm{U}-13\left(\mathrm{VO}_{2}\right.$ max estimated: $46.01 \pm 2.73 \mathrm{ml} / \mathrm{kg} / \mathrm{min}$; height: $156.2 \pm$ $9.40 \mathrm{~cm}$; weight: $46.5 \pm 8.37 \mathrm{~kg})$ and $16 \mathrm{U}-14\left(\mathrm{VO}_{2} \max\right.$ : $47.91 \pm 2.43 \mathrm{ml} / \mathrm{kg} / \mathrm{min}$; height: $166.8 \pm 7.34 \mathrm{~cm}$; weight: $55.9 \pm 8.52 \mathrm{~kg}$ ) participated in this study. Goalkeepers were not evaluated, as previously reported in the literature on SSG $[9,10]$. The players belonged to a team that participated in national competitions and presented no injuries at the moment of the study. Their regular training week comprised 5 training sessions (1.5 hour each) and 1 official match. All players and their legal guardians were informed about the research procedures.

\section{Procedures}

Data collection was performed in the following 3 stages (Figure 1).

\section{Team composition}

Previous studies showed that team composition criteria might influence players' behaviours during SSG [7]. It was also demonstrated that balancing teams in accordance with players' tactical skills increased tac-

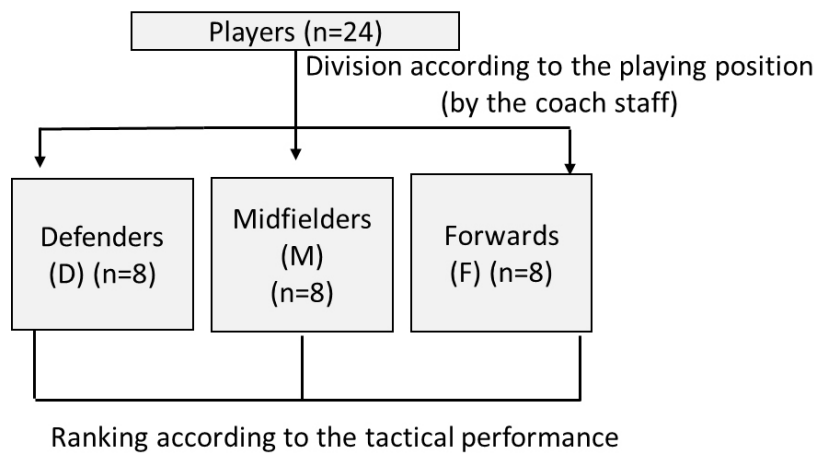

tical performance during SSG. Therefore, to compose teams balanced for players' tactical skills [7], FUT-SAT test was performed [18] in order to rank players depending on their tactical skills prior to data collection (SSG). The FUT-SAT test is a 4 -minute 3 vs. 3 game played in a $36 \times 27$ meters field, with regular goals and all rules of the formal game, including the offside.

At the beginning of the study, $24 \mathrm{U}-13$ players and 24 U-14 players were selected. Firstly, as shown at the top of Figure 1 (which represents the team composition procedures within each category), they were split into 3 groups depending on their playing positions (defenders, midfielders, and forwards), with the support of the club staff. Within each playing position, 6 players were randomly selected to play the field test against each other (6 players of the same playing position). The other 2 players of each playing position performed an additional field test against each other (6 players, 2 of each playing position). The percentage of successful tactical principles for each athlete was analysed by trained experts in the test, who ranked players according to this variable (ranking shown at the bottom of Figure 1). The top 2 and the bottom 2 athletes of each playing position were selected to be part of the study and formed 4 balanced teams (A, B, $\mathrm{C}$, and D) within each category (U-13 and U-14). The excluded midfielders (the last 4 players in the rank: $\mathrm{m}^{3}, \mathrm{~m}^{4}, \mathrm{~m}^{5}$, and $\mathrm{m}^{6}$ ) were selected to be the floaters (not evaluated) during the SSG. In order to reduce the influence of the opponents on players' behaviours, team A played against team B and team C played against team D during the whole study. Each confrontation occurred once for each SSG format, totalizing 8 SSGs and 32 4-minute bouts (16 per format).

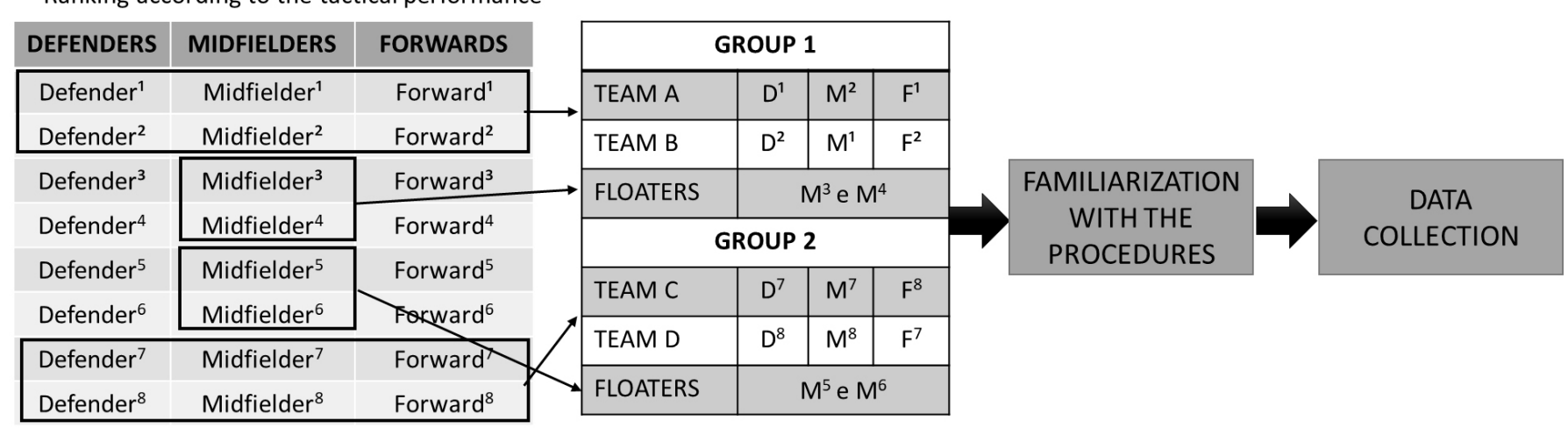

Superscript Arabic numbers indicate the ranking of each player within each position in the FUT-SAT test.

Figure 1. Team composition procedures 


\section{Familiarization with the procedures}

When the procedures for team composition were completed, the players were introduced to the two SSG formats in a familiarization session. They were considered familiarized when they did not report any additional questions.

\section{Data collection}

After a minimum interval of 24 hours, the teams performed the SSG formats. Each session started with a 10-minute preparatory activity including actions with and without the ball. Then, the first SSG bout was immediately started. During the rest inter- vals between bouts, players could drink water ad libitum. All the SSGs comprised 4 bouts of 4 minutes, with 4 minutes of passive recovery in-between. SSGs were played in a $36 \times 27$ field with all rules of the formal game (including offside). Extra balls were placed around the field to allow a quick restart of the game in case the ball went out of bounds; 7-a-side soccer goals $(6 \times 2 \mathrm{~m})$ were used. Verbal encouragement was allowed to the researcher, but no technical or tactical instructions could be provided. The experimental design comprised 2 SSG formats with floaters: 1-floater (Figure 2) SSG, in which the floater played for both teams (always supporting the team in attack), and 2-floater SSG (Figure 3), in which each team had its own floater, who was demanded to re-

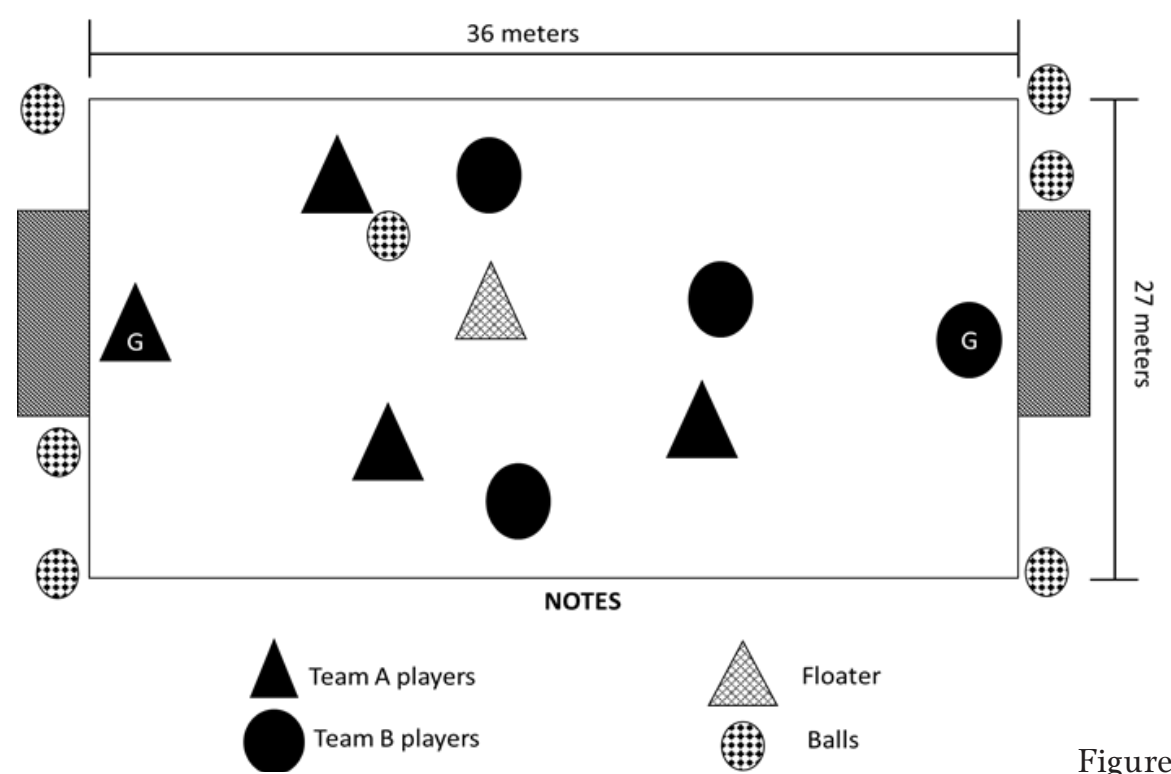

Figure 2. The 1-floater small-sided game format
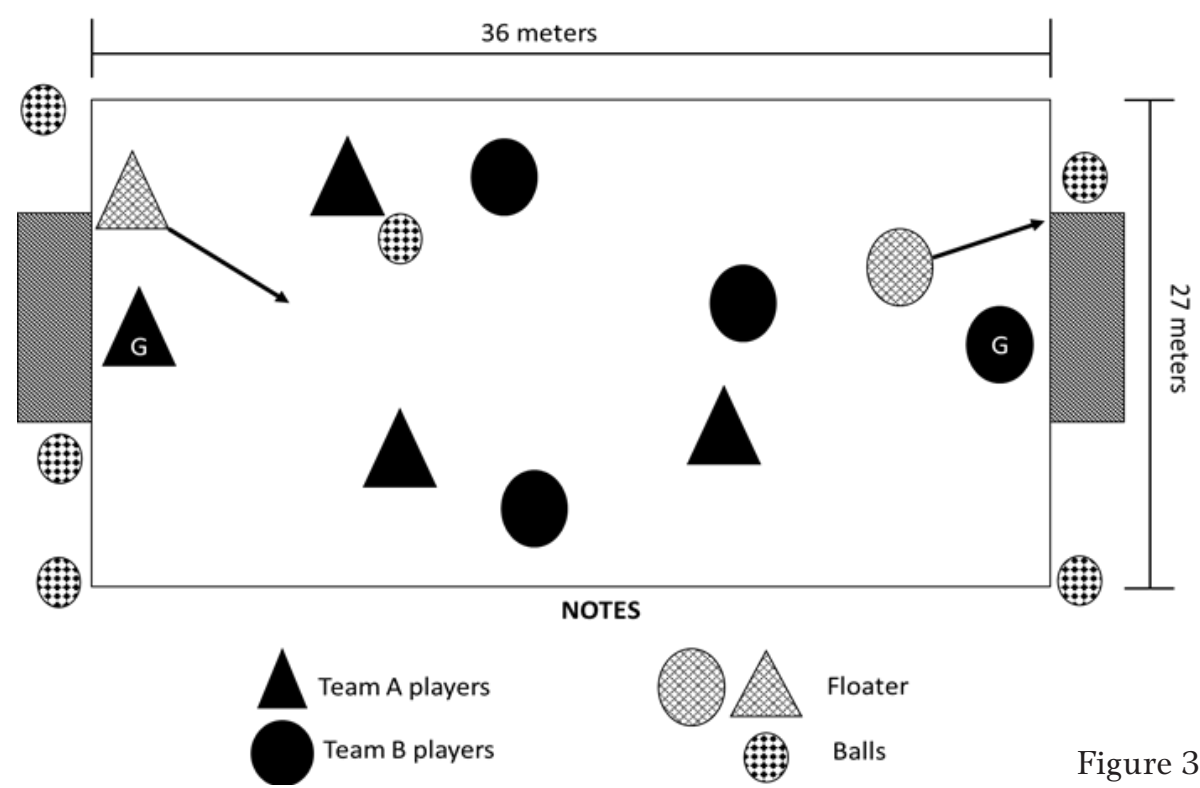

Figure 3. The 2-floater small-sided game format 
turn to the space next to their own goal every time the ball was lost by the team. In this format, the floaters' participation in the game was alternated, so just 1 floater was inside the pitch (from the team in offense), while the other one (from the team in defence) was not allowed to participate at the same moment.

\section{Measures}

\section{FUT-SAT}

The FUT-SAT consists of the observation of the core tactical principles performed by players during the game [18]. Six principles are related to the offensive phase (penetration, offensive coverage, width and length with and without the ball, depth mobility, and offensive unity) and six to the defensive phase (delay, defensive coverage, defensive balance, defensive recovery, concentration, and defensive unity). Two expert analysts (who were trained for using FUT-SAT and presented reliability above 90\%) examined the videos and recorded the frequency of actions related to each principle performed by each athlete during SSG, using the Soccer Analyser ${ }^{\circledR}$ software. The software allows the insertion of a field diagram upon the video image and establishes the game centre and ball line, which are the references adopted for the definition of the tactical principles. The dependent variables were the frequencies of each fundamental tactical principle. Every bout was recorded with a digital camera (JVC HD Everio GZ-HD520), placed in a high position that allowed the view of the whole field without changing its position.

\section{GPS devices}

The players wore a harness with a GPS device positioned between their shoulder blades. The GPS units (SPI ProX, GPSports, Canberra, Australia) were used to collect the players' physical responses in each SSG bout. Data were collected at $5 \mathrm{~Hz}$ (interpolated to $15 \mathrm{~Hz}$ ). The Team AMS R1 2016 software was used to extract the data. The reliability of the device for measuring distances in field-based movements was previously stablished in the literature and considered acceptable [19]. Four variables were analysed: total distance covered per bout (in meters) and the percentage of the total distance covered in 3 speed zones: 0-6.9 km/h (\%D 0-6.9), 6.9-14.3 km/h (\%D 6.914.3), and $14.3-21.4 \mathrm{~km} / \mathrm{h}(\% \mathrm{D} 14.3-21.4)$.

\section{Heart rate monitors}

Heart rate $(\mathrm{HR})$ was recorded at $1 \mathrm{~Hz}$ by $\mathrm{HR}$ monitors (Polar ${ }^{\circledR}$ FS1, Kempele, Finland) compatible with the GPS interface during the SSG. HR data from the recovery periods were excluded from analysis. The dependent variables were peak $\mathrm{HR}$ - the highest $\mathrm{HR}$ value recorded during the SSG - and mean $H R$ - the mean of all values of HR recorded during each bout.

\section{Data analysis}

All statistical analyses, except for effect size, were carried out by using the SPSS statistical analysis software (SPSS version 19.0 for Windows, SPSS Inc., Chicago, USA). Data are reported as means \pm standard deviations. Assumptions of normality (ShapiroWilk's test) and homoscedasticity (Levene's test) were confirmed for all variables. A two-way ANOVA (SSG format $\times$ playing position) was carried out to compare means between protocols (2 levels) and playing positions (3 levels). The Bonferroni's post-hoc test was used to make multiple paired comparisons and identify the significant differences. Cohen's $d$ effect sizes were calculated for comparisons between formats and classified as small $(d=0.2)$, moderate $(d=0.5)$, or large $(d=0.8)$. The effect size $f$ was calculated for comparisons between playing positions and classified as small $(f=0.10)$, medium $(f=0.25)$, or large $(f=0.40)$ [20]. Statistical significance was set at $p<0.05$.

\section{Data quality}

To ensure the quality of the observations, $12.5 \%$ of the SSG bouts were reanalysed by the same observers (intra-observer concordance) and by different observers (inter-observer concordance). These reanalyses occurred 21 days after the first ones. Kappa's coefficient values were higher than 0.8 for all variables. For this reason, the agreement was classified as perfect [21].

\section{Ethical approval}

The research related to human use has complied with all the relevant national regulations and institutional policies, has followed the tenets of the Declaration of Helsinki, and has been approved by the ethics committee of the Universidade Federal de Minas Gerais (CAAE: 64639417.0.0000.5149).

\section{Informed consent}

Informed consent has been obtained from all individuals included in this study and their legal guardians. 


\section{Results}

There were no interactions between the main factors of SSG format and playing position $(p>0.05)$. Therefore, results regarding each main factor will be presented independently.

Table 1 includes the comparisons between SSG formats for the frequencies of defensive and offensive tactical principles.

As shown in Table 1, the 2-floater SSG presented higher frequencies of delay ( $p=0.019$; small-to-moderate effect), defensive balance ( $p=0.009$; small-tomoderate effect), and width and length without ball ( $p=0.008$; small-to-moderate effect).

Table 2 presents the comparisons of physical and physiological variables between the SSG formats. There were no differences between the SSG formats for these variables.

Table 3 shows the comparisons between the 3 playing positions for the frequencies of each tactical prin- ciple. There were no differences between playing positions for the defensive principles. In turn, defenders performed a lower frequency of penetration actions than midfielders and forwards ( $p=0.002$; mediumto-large effect). In addition, midfielders performed a higher frequency of offensive coverage ( $p=0.001$; medium-to-large effect) and a lower frequency of width and length without ball ( $p=0.001$; medium-to-large effect) than defenders and forwards.

Table 4 presents the comparisons between the 3 playing positions for the physical and physiological variables. Midfielders presented a higher peak HR compared with forwards ( $p=0.006$; small-to-medium effect) and higher values for \%D 6.9-14.3 than defenders ( $p=0.022$; small-to-medium effect). Forwards presented lower mean HR than all other playing positions ( $p=0.001$; medium-to-large effect) and higher values for $\% \mathrm{D}$ 14.3-21.4 than midfielders.

Table 1. Means (standard deviations) of the frequency of tactical principles performed in the 1-floater and 2-floater SSG

\begin{tabular}{lcccc}
\hline Principles & 1-floater SSG & 2-floater SSG & $p$ value & Effect size \\
\hline Defensive principles & & & & \\
Delay & $5.9(2.7)$ & $7.0(3.2)$ & $0.01^{*}$ & 0.37 (small-to-moderate) \\
Defensive coverage & $1.7(1.5)$ & $1.8(1.5)$ & 0.61 & 0.06 (small) \\
Defensive balance & $6.3(3.1)$ & $7.6(3.7)$ & $0.01^{*}$ & 0.37 (small-to-moderate) \\
Defensive recovery & $2.8(2.3)$ & $2.5(1.7)$ & 0.22 & 0.14 (small) \\
Concentration & $4.2(2.7)$ & $4.0(2.3)$ & 0.60 & 0.07 (small) \\
Defensive unity & $10.6(3.9)$ & $10.1(3.9)$ & 0.40 & 0.12 (small) \\
\hline Offensive principles & & & & 0.15 (small) \\
Penetration & $3.4(1.8)$ & $3.1(2.0)$ & 0.31 & 0.10 (small) \\
Offensive coverage & $5.6(2.7)$ & $5.9(3.0)$ & 0.39 & 0.39 (small-to-moderate) \\
Width and length without ball & $11.0(4.5)$ & $12.8(4.7)$ & $0.01^{*}$ & 0.26 (small-to-moderate) \\
Width and length with ball & $1.3(0.9)$ & $1.6(1.3)$ & 0.05 & 0.17 (small) \\
Depth mobility & $2.2(1.4)$ & $2.5(1.9)$ & 0.21 & 0.21 (small-to-moderate) \\
Offensive unity & $6.0(3.4)$ & $5.3(3.0)$ & 0.16 & 0.16 \\
\hline
\end{tabular}

SSG - small-sided game

* significant difference

Table 2. Means (standard deviations) of the physical and physiological responses in the 1-floater and 2-floater SSG

\begin{tabular}{lcccc}
\hline Responses & 1-floater SSG & 2-floater SSG & $p$ value & Effect size \\
\hline Peak heart rate $(\mathrm{bpm})$ & $183.8(10.8)$ & $186.5(8.7)$ & 0.05 & 0.27 (small-to-moderate) \\
Mean heart rate (bpm) & $166.0(11.7)$ & $169.3(16.4)$ & 0.10 & 0.22 (small-to-moderate) \\
Total distance (m) & $447.7(45.3)$ & $440.5(65.4)$ & 0.37 & 0.12 (small) \\
$\% \mathrm{D} 0-6.9 \mathrm{~km} / \mathrm{h}$ & $0.409(0.07)$ & $0.414(0.07)$ & 0.65 & 0.07 (small) \\
$\% \mathrm{D} 6.9-14.3 \mathrm{~km} / \mathrm{h}$ & $0.445(0.06)$ & $0.456(0.06)$ & 0.24 & 0.18 (small) \\
$\% \mathrm{D} 14.3-21.4 \mathrm{~km} / \mathrm{h}$ & $0.135(0.04)$ & $0.124(0.04)$ & 0.09 & 0.27 (small-to-moderate) \\
\hline
\end{tabular}

SSG - small-sided game 
G. Praça et al., Players' behaviour in soccer small-sided games

Table 3. Means (standard deviations) of the frequency of tactical principles performed by defenders, midfielders, and forwards during SSG

\begin{tabular}{|c|c|c|c|c|c|c|}
\hline Principles & Defenders (1) & Midfielders (2) & Forwards (3) & $p$ value & $\mathrm{PC}$ & Effect size \\
\hline \multicolumn{7}{|l|}{ Defensive principles } \\
\hline Delay & $6.5(3.3)$ & $7.0(2.8)$ & $5.9(2.8)$ & 0.11 & & 0.36 (medium-to-large) \\
\hline Defensive coverage & $1.9(1.4)$ & $1.6(1.6)$ & $1.7(1.4)$ & 0.53 & & 0.06 (small) \\
\hline Defensive balance & $7.0(3.5)$ & $6.2(3.2)$ & $7.6(3.6)$ & 0.08 & & 0.37 (medium-to-large) \\
\hline Defensive recovery & $2.2(1.9)$ & $3.0(1.9)$ & $2.8(2.2)$ & 0.11 & & 0.14 (small-to-medium) \\
\hline Concentration & $4.0(2.1)$ & $4.3(2.9)$ & $4.1(2.4)$ & 0.78 & & 0.07 (small) \\
\hline Defensive unity & $10.6(4.3)$ & $10.3(3.9)$ & $10.2(3.5)$ & 0.86 & & 0.12 (small-to-medium) \\
\hline \multicolumn{7}{|l|}{ Offensive principles } \\
\hline Penetration & $2.6(1.6)$ & $3.7(2.2)$ & $3.4(1.6)$ & $0.01^{*}$ & $1<3,2$ & 0.25 (medium-to-large) \\
\hline Offensive coverage & $5.1(2.7)$ & $7.0(2.8)$ & $5.1(2.7)$ & $0.01^{*}$ & $2>1,3$ & 0.32 (medium-to-large) \\
\hline Width and length without ball & $12.8(4.3)$ & $10.0(4.8)$ & $12.8(4.3)$ & $0.01^{*}$ & $2<1,3$ & 0.29 (medium-to-large) \\
\hline Width and length with ball & $1.6(1.1)$ & $1.5(1.0)$ & $1.3(1.2)$ & 0.45 & & 0.11 (small-to-medium) \\
\hline Depth mobility & $2.3(1.7)$ & $2.1(1.5)$ & $2.6(1.7)$ & 0.27 & & 0.12 (small-to-medium) \\
\hline Offensive unity & $5.9(3.4)$ & $6.0(3.3)$ & $5.1(2.9)$ & 0.24 & & 0.12 (small-to-medium) \\
\hline
\end{tabular}

SSG - small-sided game, PC - pairwise comparison

* significant difference

Table 4. Means (standard deviations) of the physical demands and physiological responses of defenders, midfielders, and forwards during SSG

\begin{tabular}{lclllll}
\hline Responses & Defenders (1) & Midfielders (2) & Forwards (3) & $p$ value & PC & Effect size \\
\hline Maximum heart rate (bpm) & $185.8(8.1)$ & $187.5(7.9)$ & $182.1(12.3)$ & $0.01^{*}$ & $2>3$ & 0.23 (small-to-medium) \\
Average heart rate (bpm) & $169.2(10.2)$ & $171.4(10.8)$ & $162.3(18.7)$ & $0.01^{*}$ & $3<1,2$ & 0.29 (medium-to-large) \\
Total distance (m) & $434.8(59.4)$ & $448.3(55.5)$ & $449.3(52.8)$ & 0.27 & & 0.11 (small-to-medium) \\
$\%$ D $-6.9 \mathrm{~km} / \mathrm{h}$ & $0.427(0.08)$ & $0.409(0.07)$ & $0.400(0.06)$ & 0.10 & & 0.16 (small-to-medium) \\
$\% \mathrm{D} 6.9-14.3 \mathrm{~km} / \mathrm{h}$ & $0.435(0.06)$ & $0.468(0.06)$ & $0.448(0.06)$ & $0.02^{*}$ & $2>1$ & 0.22 (small-to-medium) \\
\%D 14.3-21.4 km/h & $0.129(0.04)$ & $0.118(0.05)$ & $0.142(0.04)$ & $0.01^{*}$ & $3>2$ & 0.24 (small-to-medium) \\
\hline
\end{tabular}

SSG - small-sided game, PC - pairwise comparison

* significant difference

\section{Discussion}

This study aimed to compare the tactical behaviour and physical and physiological variables between 1-floater and 2-floater soccer SSG in accordance with playing positions (i.e., defenders, midfielders, and forwards). Regarding the comparisons between SSG formats, we hypothesized that the 2-floater format would lead to a decrease in the physical and physiological demands and to an increase in the frequency of actions of width and length. These hypotheses were partially confirmed since differences in the frequencies of width and length tactical principles occurred but were not followed by changes in the physical and physiological demands. It was also hypothesized that midfielders' effective participation would be higher than that of the other players, leading to increased physical and physiological responses and a higher participa- tion in the game centre. These hypotheses were fully confirmed since a higher frequency of offensive coverage actions, as well as physical and physiological responses were observed for midfielders. To the best of our knowledge, no previous studies have investigated the 2-floater condition in the same way as we did in this article; thus, we reinforce the novelty of the present study and the contribution of the results to the training process of soccer players.

\section{Physical and physiological responses}

Considering the physical demands of SSG, previous studies that compared physical variables between SSG formats with and without floaters showed a decrease in the physical and physiological demands [8]. We expected that the SSG with more floaters would present a lower physical demand, which was not ob- 
served. In the present study, the area per player was kept constant during the two experimental tasks (because of floaters' alternated participation in the 2 -floater SSG), which possibly resulted in no differences in the physical demands [22].

The literature reports that midfielders usually cover higher distances than defenders and forwards [23], as well as present higher physiological responses [24]. In official matches, this result can be explained by their concomitant defensive and offensive responsibilities, which demand displacements within longer distances, despite a smaller number of sprints [23]. Although few studies have investigated the influence of playing position on players' physical responses during SSG, previous results suggest that players may act irrespective of their positional role within the team in smaller game formats, which leads to no differences in their perceived exertion during SSG [25]. On the contrary, the present study has shown that midfielders are more demanded in both physical and physiological aspects during SSG. We suggest that the presence of floaters, which increased the search for team's collective play in both offensive and defensive phases (in detriment of 1 vs. 1 actions), increased the players' functional specialization during the SSG. In this sense, a non-homogeneous role distribution, similar to that observed in official matches, could be noticed in the SSG investigated in this study. This suggests a potential use of SSG with floaters to promote more specific stimuli to players of different playing positions. Future studies should investigate this influence in SSG with and without floaters to test this hypothesis.

\section{Tactical behaviour}

As for the tactical variables, the 2-floater SSG led to a higher frequency of delays in comparison with the 1-floater format. This result can be explained by the floaters' position on the field in the 2-floater format. In this SSG, floaters were usually behind the ball line, which could encourage the defenders to try to recover the ball by duelling with the opponent. Considering that previous studies suggest that an increase in the number of players may hinder the defensive system to establish adequate duels, the presence of the floater behind the ball line in the 2-floater format could reduce the number of players that the defensive system was demanded to deal with, so a reduction in the number of delays was expected [26]. Besides, an increase in the frequency of actions of width and length without the ball (and, consequently, the compensative defensive behaviour of defensive balance) can be explained by the supportive role of the floaters for building the attack in the 2-floater SSG. Therefore, players in attack could search for more in-depth and in-length available spaces to provoke unbalances in the defensive team. This rationale is in line with a previous study in which a more in-length displacement was observed in SSG with floaters in the sidelines [11].

Finally, midfielders and forwards performed more penetration actions compared with defenders, which characterizes the progression of the ball over the game field [18]. This result is in line with the reported higher values of dribbles, shooting, and key passes for midfielders and forwards in comparison with defenders [14]. These penetration actions may have also contributed to the increase in the players' physical response since a direct intense confrontation against the defender is required. Additionally, midfielders also performed a higher frequency of offensive coverage actions. This corroborates a previous study that showed a higher frequency of both successful passing and reception for midfielders than the other playing positions during official matches [27]. These results reinforce the role of midfielders as key players for building the attack [28] and controlling the pitch centre through effective inter-player spacing [29]. In summary, our results indicate that the tactical actions performed during SSG with floaters are position-dependent, as previously demonstrated for technical actions in official matches [27].

\section{Conclusions}

We conclude that different types of floaters' participation during SSG impact players' tactical behaviour, but not their physical or physiological responses. Specifically, the 2-floater format promotes higher indepth and in-length space exploration in comparison with the 1-floater format. We also reported that tactical, physical, and physiological responses were position-dependent in SSG with floaters, with higher physical and physiological responses from midfielders, the most active players for supporting the offensive phase.

This study results address important issues regarding the physical and tactical demands of SSG, which may help coaches to choose training contents in accordance with their aims. We suggest that the 2 -floater format is more suitable for developing a tactical sense of supported progression with the ball, based on the higher number of the width and length actions. On the other hand, the 1-floater format seems more adequate to encourage transition tactics since the floater position is unpredictable. Besides, tactical, physical, 
and physiological variables are position-dependent during SSG with floaters. Therefore, the same SSG may impact players' tactical and physical responses in different ways, addressing playing position specificities. For this reason, we recommend including periodic assessments of physical and tactical development to regularly adjust training contents to players' responses. Since SSG may be used by groups with different levels - like collegiate students [30] - future studies with the formats presented here are demanded to check if the current results are applicable irrespective of the players' level of practice. Also, mental fatigue has been shown to impair players' tactical performance in SSG [31]. At this point, since the 2 -floater condition may be a novel context for many players, further studies are required to verify if players mentally fatigued are able to explore the space within such a context. Finally, as the main goal of the current article was to investigate the game, not necessarily the players within it, we recommend future studies in which players would be examined in different contexts (such as scholars, recreational players, adults) in order to identify if the results reported in the current article are applicable to other samples.

\section{Acknowledgements}

We are thankful to the Minas Gerais State Foundation for Research Support (FAPEMIG, Fundação de Amparo à Pesquisa do Estado de Minas Gerais), the Pró-reitoria de pesquisa of Universidade Federal de Minas Gerais, and the Coordination for the Improvement of Higher Education Personnel (CAPES, Coordenação de Aperfeiçoamento de Pessoal de Nível Superior) for the financial support.

\section{Disclosure statement}

No author has any financial interest or received any financial benefit from this research.

\section{Conflict of interest}

The authors state no conflict of interest.

\section{References}

1. Torrents C, Ric A, Hristovski R, Torres-Ronda L, Vicente E, Sampaio J. Emergence of exploratory, technical and tactical behavior in small-sided soccer games when manipulating the number of teammates and opponents. PLoS One. 2016;11(12):e0168866; doi: 10.1371/ journal.pone.0168866.

2. Menuchi MRTP, Moro ARP, Ambrósio PE, Pariente CAB, Araújo D. Effects of spatiotemporal constraints and age on the interactions of soccer players when competing for ball possession. J Sports Sci Med. 2018;17(3):379-391.
3. Joo CH, Hwang-Bo K, Jee H. Technical and physical activities of small-sided games in young Korean soccer players. J Strength Cond Res. 2016;30(8):2164-2173; doi: 10.1519/JSC.0000000000001319.

4. Aguiar M, Gonçalves B, Botelho G, Lemmink K, Sampaio J. Footballers' movement behaviour during 2-, 3-, 4 - and 5-a-side small-sided games. J Sports Sci. 2015; 33(12):1259-1266; doi: 10.1080/02640414.2015.102 2571.

5. Castellano J, Silva P, Usabiaga O, Barreira D. The influence of scoring targets and outer-floaters on attacking and defending team dispersion, shape and creation of space during small-sided soccer games. J Hum Kinet. 2016;51:153-163; doi: 10.1515/hukin-2015-0178.

6. Praça GM, Clemente FM, Andrade AGP, Morales JCP, Greco PJ. Network analysis in small-sided and conditioned soccer games: the influence of additional players and playing position. Kinesiology. 2017;49(2):185193; doi: 10.26582/k.49.2.8.

7. Praça GM, Morales JCP, Moreira PED, Peixoto GHC, Bredt ST, Chagas MH, et al. Tactical behavior in soccer small-sided games: influence of team composition criteria. Rev Bras Cineantropom Desempenho Hum. 2017;19(3):354-363; doi: 10.5007/1980-0037.2017v1 9n3p354.

8. Hill-Haas SV, Coutts AJ, Dawson BT, Rowsell GJ. Timemotion characteristics and physiological responses of small-sided games in elite youth players: the influence of player number and rule changes. J Strength Cond Res. 2010;24(8):2149-2156; doi: 10.1519/JSC.0b013e3181 af5265.

9. Padilha MB, Guilherme J, Serra-Olivares J, Roca A, Teoldo I. The influence of floaters on players' tactical behaviour in small-sided and conditioned soccer games. Int J Perform Anal Sport. 2017;17(5):721-736; doi: 10.1080/24748668.2017.1390723.

10. Praça GM, Bredt SGT, Torres JO, Custódio IJO, Andrade AGP, Morales JCP, et al. Influence of numerical superiority and players' tactical knowledge on perceived exertion and physical and physiological demands in soccer small-sided games. Rev Psicol Deporte. 2018; 27(2):29-36.

11. Praça GM, Folgado H, Andrade AGP, Greco PJ. Influence of additional players on collective tactical behavior in small-sided soccer games. Rev Bras Cineantropom Desempenho Hum. 2016;18(1):62-71; doi: 10.5007/ 1980-0037.2016v18n1p62.

12. Lacome M, Simpson BM, Cholley Y, Buchheit M. Locomotor and heart rate responses of floaters during small-sided games in elite soccer players: effect of pitch size and inclusion of goalkeepers. Int J Sports Physiol Perform. 2018;13(5):668-671; doi: 10.1123/ijspp.20170340 .

13. Barreira D, Garganta J, Castellano J, Anguera MT. SoccerEye: a software solution to observe and record behaviours in sport settings. Open Sports Sci J. 2013; 6:47-55; doi: 10.2174/1875399X01306010047. 
14. Yi Q, Jia H, Liu H, Gómez MÁ. Technical demands of different playing positions in the UEFA Champions League. Int J Perform Anal Sport. 2018;18(6):926-937; doi: 10.1080/24748668.2018.1528524.

15. Mallo J, Mena E, Nevado F, Paredes V. Physical demands of top-class soccer friendly matches in relation to a playing position using global positioning system technology. J Hum Kinet. 2015;47:179-188; doi: 10.1515/ hukin-2015-0073.

16. Sánchez-Sánchez J, Hernández D, Casamichana D, Martínez-Salazar C, Ramirez-Campillo R, Sampaio J. Heart rate, technical performance, and session-RPE in elite youth soccer small-sided games played with wildcard players. J Strength Cond Res. 2017;31(10):26782685; doi: 10.1519/JSC.0000000000001736.

17. Clemente FM, Sarmento H, Rabbani A, Van Der Linden CMI, Kargarfard M, Costa IT. Variations of external load variables between medium- and large-sided soccer games in professional players. Res Sports Med. 2019;27(1):50-59; doi:10.1080/15438627.2018.1511560.

18. Costa IT, Garganta J, Greco PJ, Mesquita I, Maia J. System of tactical assessment in soccer (FUT-SAT): development and preliminary validation. Motricidade. 2011;7(1):69-83.

19. Köklü Y, Arslan Y, Alemdaroğlu U, Duffield R. Accuracy and reliability of SPI ProX global positioning system devices for measuring movement demands of team sports. J Sports Med Phys Fitness. 2015;55(5):471-477.

20. Cohen J. Statistical power analysis for the behavioral sciences. New York: Lawrence Erlbaum Associates; 1988.

21. Robinson G, O’Donoghue PG. A weighted kappa statistic for reliability testing in performance analysis of sport. Int J Perform Anal Sport. 2007;7(1):12-19; doi: 10.1080/24748668.2007.11868383.

22. Sarmento H, Clemente FM, Harper LD, Costa IT, Owen A, Figueiredo AJ. Small sided games in soccer - a systematic review. Int J Perform Anal Sport. 2018; 18(5):693-749; doi:10.1080/24748668.2018.1517288.

23. Rivilla-García J, Calvo LC, Jiménez-Rubio S, Paredes-Hernández V, Muñoz A, van den Tillaar R, et al. Characteristics of very high intensity runs of soccer players in relation to their playing position and playing half in the 2013-14 Spanish La Liga season. J Hum Kinet. 2019;66:213-222; doi: 10.2478/hukin-20180058.

24. Dellal A, Owen AL, Wong DP, Krustrup P, van Exsel M, Mallo J. Technical and physical demands of small vs. large sided games in relation to playing position in elite soccer. Hum Mov Sci. 2012;31(4):957-969; doi: 10.1016/j.humov.2011.08.013.

25. Owen AL, Dunlop G, Rouissi M, Haddad M, Mendes B, Chamari K. Analysis of positional training loads (ratings of perceived exertion) during various-sided games in European professional soccer players. Int J Sports Sci Coach. 2016;11(3):374-381; doi: 10.1177/174795411 6644064 .
26. Silva B, Garganta J, Santos R, Teoldo I. Comparing tactical behaviour of soccer players in 3 vs. 3 and 6 vs. 6 small-sided games. J Hum Kinet. 2014;41:191-202; doi: 10.2478/hukin-2014-0047.

27. Brito Â, Roriz P, Silva P, Duarte R, Garganta J. Effects of pitch surface and playing position on external load activity profiles and technical demands of young soccer players in match play. Int J Perform Anal Sport. 2017;17(6):902-918; doi: 10.1080/24748668.2017. 1407207.

28. Clemente FM, Martins FML, Kalamaras D, Wong DP, Mendes RS. Midfielder as the prominent participant in the building attack: a network analysis of national teams in FIFA World Cup 2014. Int J Perform Anal Sport. 2015;15(2):704-722; doi: 10.1080/24748668. 2015.11868825.

29. Gonçalves BV, Figueira BE, Maçãs V, Sampaio J. Effect of player position on movement behaviour, physical and physiological performances during an 11-aside football game. J Sport Sci. 2014;32(2):191-199; doi: 10.1080/02640414.2013.816761.

30. Clemente FM, Nikolaidis PT, Van Der Linden CMIN, Silva B. Effects of small-sided soccer games on internal and external load and lower limb power: a pilot study in collegiate players. Hum Mov. 2017;18(1):5057; doi: 10.1515/humo-2017-0007.

31. Kunrath CA, Cardoso F, Nakamura FY, Teoldo I. Mental fatigue as a conditioner of the tactical and physical response in soccer players: a pilot study. Hum Mov. 2018;19(3):16-22; doi: 10.5114/hm.2018.76075. 